\title{
CARACTERISATION DE L'ORDRE PAR DIFFRACTION D'ELECTRONS
}

\author{
J.C. LE Bosséa ${ }^{a}$, J. Lopez ${ }^{a}$, G. HANSALI ${ }^{a}$, G. WiatrowSKi ${ }^{b}$ \\ ET J. Rousseau ${ }^{a}$
}

${ }^{a}$ Laboratoire de Tribologie et de Dynamique des Systémes, CNRS, URA 855

École Nationale d'Ingénieurs de St Étienne

58 rue Jean Parot, $42023 \mathrm{St}$ Étienne, France

${ }^{b}$ Laboratoire de Physique des Solides, Université de Lódź

Pomorska 149/153, 90-236 Lódź, Pologne

CIIARACTERIZATION OF THE ORDER BY ELECTRON DIFFRACTION: In the case of a solid with a local atomic order, we can consider only a statistical description of atomic positions. For a homogeneous phase, this description is given by the two-site occupancy correlation function, the three-site occupancy correlation function, ... etc. The local atomic order is investigated by experiments in which the solid is bombarded with a well-collimated monoenergetic beam of particles (here electrons). Starting from a model describing the force law between an electron and an atomic scatterer and from a model of the solid describing the distribution of atoms in space, a computation of elastically backscattered intensities can be carried out. The latter depends on geometrical parameters which are determined in such a way that one gets the best agreement between the results of calculation and the measurements. This work aims to provide the way of proceeding to calculate the diffuse intensity. The main difficulty in this task appears when we undertake to treat the problem of multiple scattering. We provide here a detailed description of a calculation corresponding to the case of the backscattering of electrons at a single crystal surface covered with a partially disordered atomic layer. Assuming that the coverage in adatoms is small and these adatoms are weak scatterers, we can neglect the multiple scattering events inside the layer. This simplifying assumption leads to an expression of the diffuse intensity in terms of: the Fourier transform on the 2D surface lattice of the pair correlation function; the renormalized transition matrices of the different kinds of adatoms.

PACS numbers: 61.14.Dc, 68.10.Jy

\section{Introduction}

Un solide peut être décrit comme un arrangement plus ou moins régulier d'atomes. Dans le cas d'un solide monocristallin, un motif constitué par un ou plusieurs atomes se répète périodiquement selon un réseau tridimentionnel. En 
réalité, la présence dans le volume du solide de défauts tels que lacunes, impurctés, dislocations ... etc. est à l'origine d'écarts par rapport à cet arrangement idéal. Ce n'est que sur des distances grandes devant la distance interatomique, mais petite devant la taille du cristal qu'un tel arrangement peut être observé. Dans le cas d'un solide polycristallin, on retrouve cette structure tripériodique presque parfaite à l'intérieur de grains dont la taille peut varier de quelques fractions de microns à quelques millimètres. Chaque grain correspond à une orientation particulière du réseau. Dans la zone limitrophe située entre les grains la densité de défauts, de dislocations en particulier, est tellement élevée que la notion de réseau s'estompe presque totalement. On parle encore d'un ordre à courte portée dans la mesure où dans cette zone, seules les positions des atomes voisins sont corrélées. Dans le cas enfin où cet ordre à courte portée n'est plus seulement confiné dans les régions limitrophes de grains, mais s'étend à tout le volume du matériau, on parle alors de solide amorphe.

Nous venons d'évoquer l'ordre atomique dans le volume du solide. Cependant pour les phénomènes se produisant à l'interface entre deux phases (catalyse hétérogène, chimisorption, adhésion, tribologie, ....etc.), l'ordre atomique dans le voisinage de la surface joue un rôle important. Cet ordre est généralement différent de l'ordre volumique, car le fait de briser des liaisons atomiques pour former une surface induit la plupart du temps une reconstruction du paysage atomique dans le voisinage de cette surface.

Les techniques expérimentales d'analyse de l'ordre atomique dans le volume ou à la surface d'un solide sont toutes basées sur le principe suivant. On bombarde l'échantillon à étudier avec un faisceau parallèle de particules (photons $\mathrm{X}$, électrons, neutrons, atomes) dont l'énergie est bien définie. La longueur d'onde de De Broglie de ces particules doit être de l'ordre de grandeur ou inféricure aux distances interatomiques dans le solide. On mesure la distribution angulaire des particules qui ont été diffusées élastiquement par l'échantillon à étudier. On répète généralement ces mesures avec des particules de différentes énergies. Il s'agit alors de tirer des résultats obtenus, des informations relatives à la structure de l'échantillon. On peut d'autre part effectuer des prédictions théoriques de la distribution angulaire des particules diffusées sur la base:

- d'un modèle décrivant l'interaction élastique particule/atome;

- d'un modèle décrivant la répartition spatiale des atomes de l'échantillon.

En agissant sur les paramètres de ces modèles, on s'efforce d'ajuster au mieux les résultats de la théorie avec ceux de l'expérience. Lors de la confrontation expérience/théorie, il ne faut jamais perdre de vue:

- que les calculs théoriques ne peuvent être menés à leur terme qu'aprés avoir fait certaines hypothèses simplificatrices dont on ne mesure pas toujours très bien l'incidence;

- que les résultats des expériences sont entachées d'erreurs que l'on a également du mal à évaluer.

Le but de cet article est d'aborder le problème du calcul des intensités de particules diffusées dans le cas des électrons. Les électrons dits rapides ont des énergies pouvant aller de 15 à $200 \mathrm{keV}$. Ils sont utilisés essentiellement pour la caractérisation de l'ordre en volume sur des échantillons de très petites tailles, 
c'est à dire dont l'épaisseur, choisie en fonction du libre parcours moyen inélastique des électrons, peut varier, selon le type de matériau et l'énergie des électrons, de quelques nanomètres à une centaine de nanomètres. On mesure donc l'intensité des électrons ayant traversés l'échantillon après avoir été diffusés élastiquement par les atomes. Les électrons dits lents ont des énergies comprises entre 30 et $300 \mathrm{eV}$. A cette énergie, leur libre parcours moyen inélastique est de l'ordre de quclques distances interatomiques. A moins d'utiliser des échantillons d'épaisseurs extrêmement faibles, ce qui est difficilement réalisable, il est hors de question de travailler en transmission, comme avec les électrons rapides. On mesure donc les électrons lents rétrodiffusés élastiquement. Ils n'ont parcouru au plus que quelques distances interatomiques à l'intérieur de la matière. Par conséquent, ils ont été diffusés par des atomes voisins de la surface. Cette technique est adaptée à l'étude de l'ordre atomique à la surface.

\section{La diffusion multiple}

Chaque atome de l'échantillon étudié est "éclairé":

- par une source principale qui est le faisceau d'électrons issu du canon à électrons;

- par des sources secondaires constituées par ses atomes voisins qui diffusent les électrons qu'ils reçoivent du canon.

Une théorie est dite cinématique si elle néglige l'effet des sources secondaires, c'est à dire les phénomènes de diffusion multiple. Dans le cas contraire, on parle de théorie dynamique.

Dans le cas des électrons rapides (d'énergie comprise entre 15 et $200 \mathrm{keV}$ ), chaque source secondaire diffuse peu d'électrons, mais le libre parcours moyen inélastique $\lambda$ des électrons est grand devant les distances interatomiques. Il varie typiquement de 10 à 100 nanomètres suivant l'énergie des électrons et le matériau étudié et il est une fonction croissante de l'énergie. Par conséquent, le nombre de sources secondaires à prendre en compte dans le voisinage d'un atome donné croit avec le libre parcours moyen. Si l'onde issue des sources secondaires et reçue par un atome est une fraction non négligeable de l'onde incidente primaire, alors le calcul de l'intensité diffusée doit être effectué en ayant recours à une théorie dynamique. Lorsque l'on fait croître l'énergie des électrons incidents, leur section efficace de diffusion élastique diminue (les atomes diffusent moins les électrons), alors que leur libre parcours moyen inélastique $\lambda$ augmente. Il y a là un phénomène de compensation: un atome quelconque est "éclairé" par un nombre de plus en plus grand de sources secondaires qui diffusent de moins en moins les électrons. Cependant, ce nombre est limité par l'épaisseur de l'échantillon. Quand elle devient inférieure à $\lambda$, on peut espérer que le recours à une théorie cinématique - plus simple - soit possible.

Dans le cas des électrons lents, le libre parcours moyen inélastique des électrons est de l'ordre de quelques distances interatomiques et ainsi le nombre de sources secondaires éclairant effectivement un atome donné est très réduit par rapport au cas précédent. Cependant, comme la section efficace de diffusion élastique d'un électron par un atome est considérablement plus grande à $150 \mathrm{eV}$ qu'à $50 \mathrm{keV}$, 
ces sources secondaires sont bien plus intenses que dans le cas des électrons rapides. Une théorie de diffraction d'électrons lents doit donc aussi être une théorie dynamique.

D'un point de vue général, cette théorie doit décrire comment un système $S$ diffuse des électrons en présence d'un autre système $L$ et réciproquement [1]. $S$ et $L$ peuvent être deux atomes et alors elle décrit la diffusion d'électrons par la molécule $S L ; S$ peut aussi être un groupe d'atomes (le substrat) et $L$ un autre groupe d'atomes (l'adsorbat) et alors elle décrit la diffusion d'électrons par le complexe adsorbat/substrat $S+L$. Nous introduirons la théorie dynamique à travers ce dernier exemple.

Notre but est de calculer l'intensité d'électrons diflusés par $S+L$, par unité d'intensité d'électrons incidents, par unité d'angle solide, dans une direction donnée. Cette quantité s'exprime évidemment à l'aide de la fonction d'onde $|\Psi\rangle$ des électrons en interaction avec la cible. Cette fonction d'onde ne peut elle-même être calculée que si l'on connait le potentiel d'interaction d'un électron avec $S+L$. Il est la somme de deux potentiels:

- l'un $V_{S}$ décrivant l'interaction d'un électron avec l'ensemble $S$ des atomes du substrat;

- l'autre $V_{L}$ décrivant l'interaction d'un électron avec l'ensemble $L$ des atomes de l'adsorbat. La fonction d'onde cherchée $|\Psi\rangle$ est solution de l'équation de Lipmann-Schwinger

$$
|\Psi\rangle=|\Phi\rangle+G_{0}^{+}(E)\left[V_{S}+V_{L}\right]|\Psi\rangle .
$$

Equation (1) fait intervenir $G_{0}^{+}(E)$, fonction de Green de l'électron libre d'énergie cinétique $E$ et la fonction d'onde $|\Phi\rangle$ à laquelle se réduit $|\Phi\rangle$ quand on annule $V_{S}$ et $V_{L}$, c'est à dire quand on retire le diffuseur $S+L$. La fonction d'onde $|\Phi\rangle$ décrit donc l'électron libre incident. On en déduit que la fonction d'onde $|\Psi\rangle$ est la somme de l'onde incidente $|\Phi\rangle$ et du second terme de (1) qui s'identifie à l'onde diffusée. Remarquons que si on ne s'interessait qu'à la diffusion par le substrat, on devrait résoudre une équation analogue à (1) obtenue en annulant $V_{L}$.

$A u$ lieu de rechercher une fonction d'onde $|\Psi\rangle$ à partir d'un opérateur potentiel connu $V_{S}+V_{L}$, on recherche un opérateur inconnu noté $T_{S+L}$ défini par

$$
\left[V_{S}+V_{L}\right]|\Psi\rangle=T_{S+L}|\Phi\rangle \text {. }
$$

Une telle matrice s'appelle matrice de transition: c'est la matrice transition du diffuseur $S+L$. Si $|\Phi\rangle$ est l'état de vecteur d'onde $k_{1}$ noté $\left|k_{1}\right\rangle$, l'amplitude de probabilité de transition de l'état de vecteur d'onde $k_{1}$ vers l'état de vecteur d'onde $k_{2} \neq k_{1}$ noté $\left|k_{2}\right\rangle$ vaut

$$
\left\langle k_{2} \mid \Psi\right\rangle=\left\langle k_{2}\left|G_{0}^{+}(E) T_{S+L}\right| k_{1}\right\rangle .
$$

Le problème est de calculer $T_{S+L}$ en fonction des matrices transition $T_{S}$ et $T_{L}$ des substrat et adsorbat isolés, définies par une relation analogue à (2). Pour cela, on réécrit (1) sous la forme des deux équations suivantes:

$$
|\Psi\rangle=\left|\varphi_{L}\right\rangle+G_{0}^{+}(E) V_{L}|\Psi\rangle
$$

avec

$$
\left|\varphi_{L}\right\rangle=|\Phi\rangle+G_{0}^{+}(E) V_{S}|\Psi\rangle .
$$


En échangeant les rôles de $L$ et $S$, on peut aussi réécrire (1) sous la forme

$$
|\Psi\rangle=\left|\varphi_{S}\right\rangle+G_{0}^{+}(E) V_{S}|\Psi\rangle
$$

avec

$$
\left|\varphi_{S}\right\rangle=|\Phi\rangle+G_{0}^{+}(E) V_{L}|\Psi\rangle
$$

L'onde $\left|\varphi_{L}\right\rangle$ est la somme de l'onde incidente $|\Phi\rangle$ et de l'onde diffusée $G_{0}^{+}(E) V_{S}|\Psi\rangle$ par le diffuseur $S$ en présence de $L$. Elle représente l'onde incidente effective arrivant sur le diffuseur $L$. Alors, Eq. (4a) exprime que l'onde totale $|\Psi\rangle$ est la somme de l'onde incidente arrivant sur $L$ et de l'onde diffusée par $L$. Par définition

$$
V_{L}|\Psi\rangle=T_{L}\left|\varphi_{L}\right\rangle
$$

et de façon analogue, nous pourrions écrire

$$
V_{S}|\Psi\rangle=T_{S}\left|\varphi_{S}\right\rangle \text {. }
$$

Au lieu de calculer les fonctions d'onde $\left|\varphi_{S}\right\rangle$ et $\left|\varphi_{L}\right\rangle$, on calcule les matrices transition effectives $T_{S}^{*}$ et $T_{L}^{*}$ définies par

$$
\begin{aligned}
& T_{S}\left|\varphi_{S}\right\rangle=T_{S}^{*}|\Phi\rangle, \\
& T_{L}\left|\varphi_{L}\right\rangle=T_{L}^{*}|\Phi\rangle .
\end{aligned}
$$

En combinant les équations précédentes, on arrive aux résultats suivants. La matrice transition $T_{S+L}$ du complexe $S+L$ est la somme des matrices transitions effectives de chaque constituant du complexe

$$
T_{S+L}=T_{S}^{*}+T_{L}^{*} \text {. }
$$

L'approximation cinématique consiste à remplacer (8) par

$$
T_{S+L}=T_{S}+T_{L}
$$

De plus, les matrices transitions effectives $T_{S}^{*}$ et $T_{L}^{*}$ sont solutions des équations suivantes:

$$
\begin{aligned}
& G_{0}^{+}(E) T_{S}^{*}=G_{0}^{+}(E) T_{S}+G_{0}^{+}(E) T_{S} G_{0}^{+}(E) T_{L}^{*}, \\
& G_{0}^{+}(E) T_{L}^{*}=G_{0}^{+}(E) T_{L}+G_{0}^{+}(E) T_{L} G_{0}^{+}(E) T_{S}^{*} .
\end{aligned}
$$

Nous avons multiplié à gauche par $G_{0}^{+}(E)$, car $G_{0}^{+}(E) T$ est une amplitude de probabilité de transition. L'interprétation physique de ces équations est évidente. Considérons par exemple (9a). $\left\langle k_{2}\left|G_{0}^{+}(E) T_{S}^{*}\right| k_{1}\right\rangle$ est l'amplitude de probabilité pour qu'un électron de vecteur d'onde $k_{1}$ soit diffusé par le diffuseur $S$ en présence $d u$ diffuseur $L$ dans l'état de vecteur d'onde $k_{2} \neq k_{1}$. Cette amplitude est la somme de deux termes. Le premier $\left\langle k_{2}\left|G_{0}^{+}(E) T_{S}\right| k_{1}\right\rangle$ est l'amplitude de probabilité pour qu'un électron de vecteur d'onde $k_{1}$ soit diffusé par le diffuseur $S$ isolé dans l'état de vecteur d'onde $k_{2}$. Le second est l'amplitude de probabilité pour qu'un processus de diffusion par le diffuseur $L$ en présence du diffuseur $S$ soit suivi d'un processus de diffusion par le diffuseur $L$ isolé, l'état initial étant $\boldsymbol{k}_{1}$ et l'état final $\boldsymbol{k}_{2}$.

Nous venons de donner, à travers l'exemple précédent, les notions essentielles sur les phénomènes de diffusion multiple. On peut appliquer ces notions à différents problèmes. Par exemple, celui de la diffusion multiple par le substrat lorsque celui-ci est un cristal parfait limité par un plan de haute densité. On considère ce cristal comme un empilement de plans atomiques ou de paquets de plans 
atomiques identiques et on procède en deux temps. On calcule d'abord la matrice amplitude de diffusion du plan ou du paquet de plans considérés. Une des diffcultés de cette méthode qui est l'évaluation de la matrice de diffusion multiple, et que nous ne définirons pas ici, a été surmontée par Kambe [2-4]. On calcule ensuite l'amplitude de diffusion d'un empilement de plans ou de paquets de plans. On peut utiliser pour cela différentes méthodes: méthode de doublement du nombre de couches [5], méthode des ondes de Bloch [6]. On verra au paragraphe suivant comment est traité le problème de la diffusion multiple dans une couche atomique désordonnée adsorbée sur un substrat ordonné.

Dans le cas des électrons rapides, la diffusion atomique se fait dans des directions très voisines de la direction du faisceau incident. En effet, dans la mesure où l'énergie d'interaction de l'électron devient petite devant sont énergie cinétique, on peut appliquet la théorie des perturbations au premier ordre et l'on constate que l'amplitude de diffusion de l'état de vecteur d'onde $k_{1}$ vers celui de vecteur d'onde $\boldsymbol{k}_{2}$ est proportionnelle à la transformée de Fourier spatiale du potentiel atomique pour le vecteur $q=k_{2}-k_{1}$. Pour des électrons rapides $k_{1}$ et $k_{2}$ sont des vecteurs de grande norme et ainsi, à moins que $k_{2}$ soit très voisin de $k_{1}$, exp(iqr) oscille très rapidement dans des zones où le potentiel $V(r)$ reste quasiment constant. La transformée de Fourier de $V(r)$ est donc négligeable à chaque fois que le vecteur d'onde $q$ n'est pas petit, c'est à dire quand le vecteur d'onde final n'est pas proche du vecteur d'onde initial. Les processus de diffusion multiple ne concernent que les atomes situés le plus en avant à l'intérieur du matériau dans la direction du faisceau incident. Les atomes situés près de la surface, côté canon, sont éclairés par des électrons qui n'ont pas été déviés par d'autres atomes. Au contraire, les atomes situés à une grande distance de la surface sont éclairés par des électrons qui ont déjà effectués un long voyage à travers la matière et qui, de ce fait, ont plus de chances d'avoir déjà subi des déviations par des atomes situés sur leur parcours. La matrice de transition effective des atomes de la cible dépend finalement de leur position à l'intérieur de la cible.

\section{Intensité diffusée par une couche partiellement ordonnée}

Celte intensité s'exprime évidemment en fonction de la partie diffusée $\left|\Psi_{\mathrm{d}}\right\rangle$ dans l'onde totale $|\Psi\rangle$. C'est donc par le calcul de cette onde que nous commencerons. Partant des relations (8) et (9), on montre sans peine que $\left|\Psi_{\mathrm{d}}\right\rangle$ est donné par l'expression

$$
\begin{aligned}
& \left|\Psi_{\mathrm{d}}\right\rangle=G_{0}^{+} T_{S}|\phi\rangle+G_{0}^{+} T_{L}|\phi\rangle \\
& +G_{0}^{+} T_{S} G_{0}^{+} T_{L}|\phi\rangle+G_{0}^{+} T_{L} G_{0}^{+} T_{S}^{*}|\phi\rangle+G_{0}^{+} T_{S} G_{0}^{+} T_{L} G_{0}^{+} T_{S}^{*}|\phi\rangle .
\end{aligned}
$$

Les cinq termes de cette expression ont une interprétation physique très simple. Le premier décrit la rétrodiffusion par le substrat seul. Celui-ci ayant une symétrie de translation parfaite (c'est un solide monocristallin, donc parfaitement ordonné), $G_{0}^{+} T_{S}|\phi\rangle$ est une onde de type Bloch. Cette onde contribue à l'intensité diffractée par le complexe $S+L$. Rappelons que le problème que nous examinons ici est celui de la caractérisation de l'ordre à courte portée dans la couche adsorbée. Or, c'est cet ordre local issu de la rupture de la symétrie de translation dans la couche qui 
est à l'origine du fond d'intensité diffusée observé entre les taches de diffraction. Le problème de l'ordre local ne nécessite donc aucunement le calcul des intensités dans les directions de diffraction et nous ne tiendrons donc pas compte du premier terme de (10).

Le second terme de (10) est l'onde rétrodiffusée par la couche adsorbée. Le troisième terme décrit un processus de diffusion multiple dans lequel un électron est transmis à travers la couche adsorbée avant d'être rétrodiffusé par le substrat. Le processus décrit par le quatrième terme est la transmission d'un électron à travers la couche adsorbée précedée par sa rétrodiffusion par le substrat en présence de l'adsorbat. Nous ferons à ce stade la première approximation consistant à remplacer dans (10) l'amplitude caractérisant cette dernière, c'est à dire $G_{0}^{+} T_{S}^{*}$, par $G_{0}^{+} T_{S}$. On peut justifier cette approximation en remarquant que les atomes adsorbés sont souvent des atomes qui diffusent assez faiblement les électrons. Ils sont vis à vis du substrat des sources secondaires peu intenses. En outre, les couches adsorbées étudiées sont des fractions de monocouche, et ces sources secondaires sont distribuées de façon éparse à la surface du substrat. L'approximation ci-dessus concerne aussi le cinquième terme décrivant un processus de diffusion multiple dans lequel un électron est rétrodiffusé par le substrat, puis par la couche et enfin par le substrat. Finalement, le calcul de l'intensité diffusée en dehors des directions de diffraction nécessite celui de la fonction d'onde [7]:

$$
\begin{aligned}
\left|\Psi_{\mathrm{d}}\right\rangle=G_{0}^{+} T_{L}|\phi\rangle+G_{0}^{+} T_{S} G_{0}^{+} T_{L}|\phi\rangle & \\
& +G_{0}^{+} T_{L} G_{0}^{+} T_{S}|\phi\rangle+G_{0}^{+} T_{S} G_{0}^{+} T_{L} G_{0}^{+} T_{S}|\phi\rangle .
\end{aligned}
$$

L'amplitude de diffusion du substrat $G_{0}^{+} T_{S}$ est calculée en utilisant les méthodes de Kambe et des ondes de Bloch évoquées au paragraphe précédent. Supposons que $|\Phi\rangle$ soit l'onde plane de vecteur d'onde $k_{1}^{+}$(le + signifie que la composante du vecteur d'ondè incident perpendiculaire à la surface est dirigée du vide vers le substrat, c'est à dire, selon nos conventions, dans le sens positif de l'axe $O z$ ). On peut montrer que [5]:

$$
\left\langle k_{2}^{-}\left|G_{0}^{+} T_{S}\right| k_{1}^{+}\right\rangle=\sum_{\{g\}} M_{S}\left(k_{1 g}^{-} \leftarrow k_{1}^{+}\right) \delta\left(k_{2}^{-}-k_{1 g}^{-}\right) .
$$

$k_{1}^{+}$et $k_{1 g}^{-}$sont donnés par

$$
\begin{aligned}
& k_{1}^{+}=k_{1 \|}+z k_{1 \perp}, \\
& k_{1 \perp}=\sqrt{E-\left(k_{1 \|}\right)^{2}}, \\
& k_{1 g}^{-}=k_{1 \|}+g-z k_{1 g \perp}, \\
& k_{1 g \perp}=\sqrt{E-\left(k_{1 \|}+g\right)^{2}} .
\end{aligned}
$$

Dans ces expressions $z$ est un vecteur normé perpendiculaire à la surface dans le sens vide substrat de l'axe $O z$ et $g$ est un vecteur du réseau réciproque associé au réseau $2 \mathrm{D}$ du substrat. Il ne nous reste plus qu'à calculer l'amplitude $G_{0}^{+} T_{L}$ de diffusion de la couche adsorbée. 


\subsection{Amplitude de diffusion de l'adsorbal}

On va appliquer les notions sur la diffusion multiple présentées au Sec. 2 dans le cas où un ou plusieurs types d'adsorbat sont réparties de façon plus ou moins aléatoire sur un ou plusieurs types de sites de chimisorption. Rappelons que pour chaque type, les sites forment un réseau identique à celui de la surface du substrat. Par exemple, pour un taux de couverture supérieur à 0.3 , une molécule de $\mathrm{CO}$ (un type d'adsorbat) peut occuper les sites top ou les 3 types de sites bridge (4 types de site au total) de la face (111) du platine. Il y a donc un réseau, et à l'intérieur de chaque maille de ce réseau plusieurs types de site. On peut aussi envisager la co-adsorption de deux types d'atomes sur un seul type de site.

Le cas d'un solide amorphe est assez différent de celui que nous traitons ici. Outre le fait que ce problème est à trois dimensions, l'absence d'un support cristallin entraine que les atomes peuvent occuper tous les points de l'espace, et non pas seulement ceux appartenant à un réseau. De plus, on n'a pas à calculer une matrice de transition de l'échantillon amorphe en présence d'un autre diffuseur. Les processus de diffusion multiple à considérer sont ceux mettant en jeu les atomes qui le composent. Ce problème de diffusion d'électrons par une substance amorphe a été abondamment étudié [8] et nous ne le traiterons pas ici. Par contre nous nous inspirerons de ce travail pour calculer la matrice de transition de la couche adsorbée.

Le potentiel de la couche adsorbée peut s'écrire

$$
V_{L}=\sum_{i, p} n_{i p} V_{i p}
$$

L'indice $i$ se réfère à une maille du réseau de surface et l'indice $p$ se réfère à un site d'adsorbtion. Rappelons qu'un site c'est un atome ou une molécule adsorbés en un point particulier de la maille du réseau $2 \mathrm{D}$. Deux sites peuvent différer, soit parce qu'ils se réfèrent à deux atomes ou molécules distincts, soit parce qu'ils se réfèrent à deux points distincts, soit pour ces deux raisons simultanément. L'équation de Lipmann-Schwinger pour la couche s'écrit

$$
|\Psi\rangle=|\phi\rangle+\sum_{i, p} n_{i p} G_{0}^{+}(E) V_{i p}|\Psi\rangle=|\phi\rangle+G_{0}^{+}(E) T_{L}|\Psi\rangle
$$

Comme nous l'avons précédemment signalé, les atomes adsorbés sont des atomes légers de petit $Z$ (H, C, O, N, ...etc.), donc de faibles diffuseurs. En outre, le taux de couverture du substrat est généralement faible. Notre deuxième hypothèse consistera à négliger les phénomènes de diffusion multiple dans la couche. Pour cela on remplace $t_{i p}^{*}$ par $t_{i p}$ dans la relation

$$
V_{i p}|\Psi\rangle=t_{i p}\left|\varphi_{i p}\right\rangle=t_{i p}^{*}|\Phi\rangle \text {. }
$$

Conformément à ce qui précède, $\left|\varphi_{i p}\right\rangle$ est l'onde incidente effective arrivant sur le site ip. La matrice de diffusion de l'adsorbat est donc donnée par

$$
G_{0}^{+}(E) T_{L}=G_{0}^{+}(E) \sum_{i, p} n_{i p} t_{i p}
$$

Reportons cette expression dans celle de $\left|\Psi_{\mathrm{d}}\right\rangle$ donnée en (11). On obtient alors

$$
\left|\Psi_{\mathrm{d}}\right\rangle=\sum_{i, p} n_{i p} K_{i p}|\Phi\rangle
$$


Dans Eq. (20a), $K_{i p}$ représente l'amplitude de diffusion du site ip:

$$
K_{i p}=G_{0}^{+} t_{i p}+G_{0}^{+} t_{i p} G_{0}^{+} T_{S}+G_{0}^{+} T_{S} G_{0}^{+} t_{i p}+G_{0}^{+} T_{S} G_{0}^{+} t_{i p} G_{0}^{+} T_{S}
$$

et $\sum_{i, p} n_{i p} K_{i p}$ est l'amplilude de diffusion de la couche adsorbée. On peut maintenant envisager de calculer la fonction d'onde $\left\langle r \mid \Psi_{\mathrm{d}}\right\rangle=\Psi_{\mathrm{d}}(r)$.

\subsection{Fonction d'onde de diffusion}

Plaçons-nous dans le cas où l'onde incidente $|\Phi\rangle$ est égal à $\left|k_{1}^{+}\right\rangle$. Après projection d'Eq. (20a) sur la base des ondes planes se transforme en.

$$
\Psi_{\mathrm{d}}(r)=\int \mathrm{d} k_{2 \|} \sum_{i, p} n_{i p} K_{i p}\left(k_{2}^{-} \leftarrow k_{1}^{+}\right) \exp \left(\mathrm{i} k_{2}^{-} r\right) .
$$

Le calcul de l'élément de matrice $K_{i p}\left(k_{2}^{-} \leftarrow k_{1}^{+}\right)$est fastidieux et nous n'en donnerons ici que les principaux jalons (pour plus de détails, voir la thèse de doctorat d'I. Zasada [9]). On calcule d'abord l'élément de matrice $\left\langle k_{2}^{-}\left|G_{0}^{+} t_{i p}\right| k_{1}^{+}\right\rangle$et on trouve

$$
\left\langle k_{2}^{-}\left|G_{0}^{+} t_{i p}\right| k_{1}^{+}\right\rangle=\frac{-\mathrm{i} \pi}{K_{2 \perp}} t_{i p}\left(k_{2}^{-} \leftarrow k_{1}^{+}\right) .
$$

$t_{i p}\left(k_{2}^{-} \leftarrow k_{1}^{+}\right)$est un élément de la matrice transition du site ip dans la base des ondes planes. Notons que

$$
K_{2 \perp}=\sqrt{E+U-\left(k_{2 \|}\right)^{2}}
$$

est la composante perpendiculaire à la surface du vecteur d'onde $k_{2}^{-}$, dans le substrat de potentiel interne $U$. En combinant Eqs. (12), (20b) et (22) on obtient

$$
K_{i p}\left(k_{2}^{-} \leftarrow k_{1}^{+}\right)=\frac{-i \pi}{K_{2 \perp}} \mathcal{I}_{i p}\left(k_{2}^{-} \leftarrow k_{1}^{+}\right)
$$

dans laquelle la matrice de transition renormalisée $\mathcal{I}_{i p}\left(k_{2}^{-} \leftarrow k_{1}^{+}\right)$du site ip a l'expression suivante [7, 10-12]:

$$
\begin{aligned}
& \mathcal{I}_{i p}\left(k_{2}^{-} \leftarrow k_{1}^{+}\right)=t_{i p}\left(k_{2}^{-} \leftarrow k_{1}^{+}\right)+\sum_{g} t_{i p}\left(k_{2}^{-} \leftarrow k_{1 g}^{-}\right) M_{S}\left(k_{1 g}^{-} \leftarrow k_{1}^{+}\right) \\
& +\sum_{g} \frac{K_{2 \perp}}{K_{2}{ }_{\perp}} M_{S}\left(k_{2}^{-} \leftarrow k_{2 g}^{+}\right) t_{i p}\left(k_{2 g}^{+} \leftarrow k_{1}^{+}\right) \\
& +\sum_{g} \sum_{g^{\prime}} \frac{K_{2 \perp}}{K_{2} \perp} M_{S}\left(k_{2}^{-} \leftarrow k_{2 g}^{+}\right) t_{i p}\left(k_{2 g}^{+} \leftarrow k_{1}^{-} g^{\prime}\right) M_{S}\left(k_{1 g^{\prime}}^{-} \leftarrow k_{1}^{+}\right) .
\end{aligned}
$$

Signalons enfin une propriété importante de la matrice $K_{i p}$ :

$$
K_{i p}\left(k_{2}^{-} \leftarrow k_{1}^{+}\right)=K_{p}\left(k_{2}^{-} \leftarrow k_{1}^{+}\right) \exp \left[-\mathrm{i}\left(k_{2 \|}-k_{1 \|}\right) \boldsymbol{R}_{i}\right] .
$$

Dans cette expression, $K_{p}$ est l'amplitude de diffusion renormalisée du site $p$ correspondant à $R_{i}=0$. Grâce à cette propriété on peut exprimer $\Psi_{\mathrm{d}}(r)$ sous une forme

$$
\Psi_{\mathrm{d}}(r)=\int \mathrm{d} k_{2 \|} \sum_{p} K_{p}\left(k_{2}^{-} \leftarrow k_{1}^{+}\right) S_{p}\left(\sigma_{2\rfloor}^{*}-k_{1 \|}\right) \exp \left(\mathrm{i} k_{2}^{-} r\right)
$$


faisant intervenir deux facteurs: $K_{p}$ qui ne dépend que des propriétés de diffusion du site $p$ et

$$
S_{p}\left(k_{2 \|}-k_{1 \|}\right)=\sum_{i} n_{i p} \exp \left[-\mathrm{i}\left(k_{2 \|}-k_{1 \|}\right) \boldsymbol{R}_{i}\right]
$$

qui ne dépend que de la distribution statistique des sites à la surface du substrat. Pour un type de site $p$, on peut définir un taux de couverture $\theta_{p}$ qui vaut $1 \mathrm{si}$ tous les sites $p$ sont occupés et 0 si aucun site n'est occupé. $N$ étant le nombre de mailles du réseau de surface, on a

$$
\begin{aligned}
& \theta_{p}=\frac{1}{N} \sum_{i=1}^{N} n_{i p}, \\
& \delta n_{i p}=n_{i p}-\theta_{p} .
\end{aligned}
$$

On reporte (29) dans l'expression de $S_{p}\left(k_{2 \|}-k_{1 \|}\right)$ et on utilisant la règle de somme

$$
\lim _{N \rightarrow \infty} \sum_{i=1}^{N} \exp \left(-\mathrm{i} \boldsymbol{q} \boldsymbol{R}_{i}\right)=\frac{4 \pi^{2}}{S_{0}} \sum_{\{\boldsymbol{g}\}} \delta(\boldsymbol{q}-\boldsymbol{g})
$$

dans laquelle $S_{0}$ est la surface de la maille élémentaire du réseau de surface. On obtient alors

$$
\begin{aligned}
& S_{p}\left(k_{2 \|}-k_{1 \|}\right)=\frac{4 \pi \theta}{S_{0}} p \sum_{\{g\}} \delta\left(k_{2 \|}-k_{1 \|}-g\right)+\delta S_{p}\left(k_{2 \|}-k_{1 \|}\right), \\
& \delta S_{p}\left(k_{2 \|}-k_{1 \|}\right)=\sum_{i} \delta n_{i p} \exp \left[-\mathrm{i}\left(k_{2 \|}-k_{1 \|}\right) R_{i}\right] .
\end{aligned}
$$

Le premier terme contribue à l'intensité diffusée dans les directions de diffraction. Ne nous interessant qu'à l'intensité diffusée en dehors de ces directions, on peut abandonner ce terme. La fonction d'onde $\Psi_{\mathrm{d}}(r)$ vaut finalement

$$
\Psi_{\mathrm{d}}(r)=\int \mathrm{d} k_{2 \|} \sum_{p} K_{p}\left(k_{2}^{-} \leftarrow k_{1}^{+}\right) \delta S_{p}\left(k_{2 \|}-k_{1 \|}\right) \exp \left(\mathrm{i} k_{2}^{-} r\right) .
$$

\section{Intensité relative rétrodiffusée par une couche désordonnée} diffusé

Il suffit de calculer le vecteur densité de courant de probabilité de présence

$$
J_{\mathrm{d}}(r)=2 \operatorname{Im}\left\{\bar{\Psi}_{\mathrm{d}}(r) \nabla \Psi_{\mathrm{d}}(r)\right\}
$$

puis le flux de ce vecteur à travers la surface $S$ de l'échantillon. L'utilisation de la relation

$$
\lim _{S \rightarrow \infty} \int_{S} \mathrm{~d}^{2} r \exp \left(\mathrm{i} q_{\|} r\right)=4 \pi^{2} \delta\left(q_{\|}\right)
$$

permet d'aboutir à l'expression suivante de ce flux:

$$
\Theta_{\mathrm{d}}=4 \pi^{2} \int \mathrm{d} k_{2 \|}\left|\sum_{p} K_{p}\left(k_{2}^{-} \leftarrow k_{1}^{+}\right) \delta S_{p}\left(k_{2 \|}-\dot{k}_{1 \|}\right)\right|^{2} k_{2 \perp} .
$$


Le flux incident $\Theta_{\mathrm{i}}=2 k_{1 \perp} S$ permet de calculer le flux relatif $\Theta_{\mathrm{d}} / \Theta_{\mathrm{i}}$. Enfin, remarquons que $\mathrm{d} k_{2 \|}=\sqrt{E} k_{2 \perp} \mathrm{d} \Omega_{2}$ s'exprime en fonction de l'élément d'angle solide $\mathrm{d} \Omega_{2}=\sin \left(\vartheta_{2}\right) \mathrm{d} \vartheta_{2} \mathrm{~d} \varphi_{2}\left(\vartheta_{2}\right.$ et $\varphi_{2}$ sont les angles des coordonnées sphériques). Cela permet d'écrire le flux relatif diffusé sous la forme

$$
\frac{\Theta_{\mathrm{d}}}{\Theta_{\mathrm{i}}}=\int \mathrm{d} \Omega_{2} \frac{\mathrm{d} I\left(k_{2}^{-} \leftarrow k_{1}^{+}\right)}{\mathrm{d} \Omega_{2}}
$$

où la quantité

$$
\frac{\mathrm{d} I\left(k_{2}^{-} \leftarrow k_{1}^{+}\right)}{\mathrm{d} \Omega_{2}}=\frac{4 \pi^{2} \sqrt{E}}{S} \frac{k_{2 \perp}^{2}}{k_{1 \perp}}\left|\sum_{p} K_{p}\left(k_{2}^{-} \leftarrow k_{1}^{+}\right) \delta S_{p}\left(k_{2 \|}-k_{1 \|}\right)\right|^{2}
$$

est le flux (ou l'intensité) relatif par unité d'angle solide dans la direction du vecteur $k_{2}^{-}$. Le calcul du module carré figurant dans $(36 \mathrm{~b})$ nous amène à calculer le produit $\overline{\delta S_{p}(q)} \delta S_{p^{\prime}}(q)$ où $q$ est la différence $k_{2 \|}-k_{1 \|}$. On montre aisément que

$$
\overline{\delta S_{p}(q)} \delta S_{p^{\prime}}(q)=N \sum_{i} \gamma_{p p^{\prime}}\left(\boldsymbol{R}_{i}\right) \exp \left(-\mathrm{i} \boldsymbol{q} \boldsymbol{R}_{i}\right)
$$

avec

$$
\gamma_{p p^{\prime}}\left(\boldsymbol{R}_{i}\right)=\frac{1}{N} \sum_{j} \delta n_{p}\left(\boldsymbol{R}_{i}+\boldsymbol{R}_{j}\right) \delta n_{p^{\prime}}\left(\boldsymbol{R}_{j}\right) .
$$

Dans Eq. (37b), les quantités du type $\delta n_{p}\left(\boldsymbol{R}_{i}\right)$ sont les fluctuations des nombres d'occupation qui ont été notées $\delta n_{i p}$ dans leur définition $(29 \mathrm{~b})$. Le sens de l'expression (37b) deviendra clair si on fait apparaître les nombres d'occupation $n_{p}\left(\boldsymbol{R}_{i}\right)$ au lieu de $\delta n_{p}\left(\boldsymbol{R}_{i}\right)$. On montre sans difficulté que

$$
\gamma_{p p^{\prime}}\left(\boldsymbol{R}_{i}\right)=\frac{1}{N} \sum_{j} n_{p}\left(\boldsymbol{R}_{i}+\boldsymbol{R}_{j}\right) n_{p^{\prime}}\left(\boldsymbol{R}_{j}\right)-\theta_{p} \theta_{p^{\prime}} .
$$

Le premier terme de (38) est la moyenne prise sur l'ensemble des sites du produit des occupations de deux sites $p$ et $p^{\prime}$ situés aux extrémités du vecteur $\boldsymbol{R}_{j}$. Comme $\theta_{p} \theta_{p^{\prime}}$ est le produit des moyennes de ces occupations, $\gamma_{p p^{\prime}}\left(\boldsymbol{R}_{i}\right)$ est une fonction de corrélation des occupations des sites $p$ et $\boldsymbol{p}^{\prime}$ extrémités de $\boldsymbol{R}_{i}$. Lorsque $\boldsymbol{R}_{i}$ est différent de 0 et que la distribution est aléatoire, $\gamma_{p p^{\prime}}\left(\boldsymbol{R}_{i}\right)=0$. Comme des sites d'adsorption de plus en plus éloignés sont de moins en moins corrélés, alors $\gamma_{p p^{\prime}}\left(\boldsymbol{R}_{i}\right) \rightarrow 0$ quand $\left\|\boldsymbol{R}_{i}\right\| \rightarrow \infty$. Examinons maintenant le cas $\boldsymbol{R}_{\boldsymbol{i}}=\mathbf{0}$. Si $\boldsymbol{p}$ et $p^{\prime}$ représentent deux adsorbats à placer sur un même site de la maille $i$, alors le produit $n_{j p} n_{j p^{\prime}}$ vaut $n_{j p} \delta_{p p^{\prime}}$ et $\gamma_{p p^{\prime}}(0)=\theta_{p} \delta_{p p^{\prime}}-\theta_{p} \theta_{p^{\prime}}$. Si $p$ et $p^{\prime}$ désignent deux sites distincts à l'intérieur d'une cellule, alors rien n'exclut qu'ils puissent simultanément être occupés, même si cela est peu probable. Dans ce cas, on ne peut donc a priori rien dire de $\gamma_{p p^{\prime}}(0)$. Exceptés dans ces cas limites, $\gamma_{p p^{\prime}}\left(\boldsymbol{R}_{i}\right)$ ne peut être connu que si l'on calcule effectivement ces moyennes. Dans l'approche présentée ici, les moyennes sont prises sur un réseau infini car on passe à la limite $N \rightarrow \infty$ à la fin des calculs. En fait, on peut transformer cette moyenne sur un ensemble de mailles par une moyenne prise sur un ensemble de configurations $[13,14]$. Si on se donne un hamiltonien modèle décrivant les interactions interadsorbats et si la couche adsorbée est à l'équilibre thermodynamique, on peut définir cet ensemble de configurations à partir des matrices densité canonique ou grand 
canonique. Comme les taux de couverture en sites $p$ occupés sont figés, on utilisera plutôt la distribution canonique.

Pour terminer ce paragraphe, introduisons comme cela se fait habituellement en théorie de la diffraction d'électrons rapides, les fonctions de corrélation de paires réduites. Pour cela, remarquons que si on écrit

$$
\begin{aligned}
\sum_{j} & n_{p}\left(\boldsymbol{R}_{i}+\boldsymbol{R}_{j}\right) n_{p^{\prime}}\left(\boldsymbol{R}_{j}\right)=\frac{\sum_{j} n_{p}\left(\boldsymbol{R}_{i}+\boldsymbol{R}_{j}\right) n_{p^{\prime}}\left(\boldsymbol{R}_{j}\right)}{\sum_{j} n_{p^{\prime}}\left(\boldsymbol{R}_{j}\right)} \sum_{j} n_{p^{\prime}}\left(\boldsymbol{R}_{j}\right) \\
= & n_{p p^{\prime}}\left(\boldsymbol{R}_{i}\right) N_{p^{\prime}},
\end{aligned}
$$

le second facteur du membre de droite représente le nombre total $N_{p^{\prime}}$ de sites $p^{\prime}$ occupés alors que le premier facteur $n_{p p^{\prime}}\left(\boldsymbol{R}_{i}\right)$ représente le nombre moyen de sites $p$ occupés à l'extrémité du vecteur $\boldsymbol{R}_{i}$ quand un site $p^{\prime}$ situé à l'origine de ce vecteur est occupé. Comme cela a été vu précédemment, dans le cas d'une couche désordonnée, l'occupation de deux sites éloignés n'est pas corrélée et par conséquent $n_{p p^{\prime}}\left(\boldsymbol{R}_{\boldsymbol{i}}\right) \rightarrow \theta_{p}$ quand $\left\|\boldsymbol{R}_{\boldsymbol{i}}\right\| \rightarrow \infty$. Il est commode d'écrire $\gamma_{p p^{\prime}}\left(\boldsymbol{R}_{\boldsymbol{i}}\right)$ sous la forme

$$
\gamma_{p p^{\prime}}\left(\boldsymbol{R}_{i}\right)=\theta_{p} \theta_{p^{\prime}}\left[\frac{n_{p p^{\prime}}\left(\boldsymbol{R}_{i}\right)}{\theta_{p}}-1\right]=\theta_{p} \theta_{p^{\prime}} g_{p p^{\prime}}\left(\boldsymbol{R}_{i}\right) .
$$

Dans cette expression, $g_{p p^{\prime}}\left(\boldsymbol{R}_{i}\right)$ est appelée fonction de corrélation de paires réduite. En reportant (40) dans (37b), on obtient

$$
\begin{aligned}
& \overline{\delta S_{p}(q)} \delta S_{p^{\prime}}(q)=N \theta_{p} \theta_{p^{\prime}} G_{p p^{\prime}}(q), \\
& G_{p p^{\prime}}(q)=\sum_{i} g_{p p^{\prime}}\left(R_{i}\right) \exp \left(-\mathrm{i} q R_{i}\right) .
\end{aligned}
$$

Revenons maintenant à l'expression (36b) de l'intensité rétrodiffusée hors canaux de diffraction. En utilisant le fait qu'à la limite $N \rightarrow \infty, S / N \rightarrow S_{0}$, aire de la maille du réseau, on arrive à l'expression finale $[13,14]$ :

$$
\begin{aligned}
& \frac{\mathrm{d} I\left(k_{2}^{-} \leftarrow k_{1}^{+}\right)}{\mathrm{d} \Omega_{2}}=\frac{4 \pi^{2} \sqrt{E}}{S} \frac{k_{2 \perp}^{2}}{k_{1 \perp}} \sum_{p, p^{\prime}} \theta_{p} \theta_{p^{\prime}} G_{p p^{\prime}}\left(k_{2 \|}-k_{1 \|}\right) \\
& \quad \times \overline{K_{p}}\left(k_{2}^{-} \leftarrow k_{1}^{+}\right) K_{p^{\prime}}\left(k_{2}^{-} \leftarrow k_{1}^{+}\right) .
\end{aligned}
$$

\section{Conclusion et discussion}

Une expression analogue à Eq. (42) est obtenue dans le traitement du cas des électrons rapides [8]. L'utilisation de cette expression dans la comparaison expérience/théorie est délicate, particulièrement dans le cas où coexistent plusieurs types de sites de chimisorption. Remarquons d'abord que dans Eq. (42), $G_{p p^{\prime}}\left(k_{2 \|}-k_{1 \|}\right)$ ne dépend que de la distribution statistique des sites $p$ et $p^{\prime}$, indépendamment de leur nature chimique (type d'atome adsorbé) et de leur structure géométrique (position des adsorbats par rapport aux atomes voisins du substrat). Par contre, $K_{p}\left(k_{2}^{-} \leftarrow k_{1}^{+}\right)$ne dépend que de la nature chimique et de la 
géométrie locale des sites de type $p$, indépendamment de leur distribution statistique à la surface. La séparation de ces deux types d'information est un problème difficile et que l'on rencontre aussi en théorie de la diffraction des électrons rapides.

Commençons par examiner la situation la plus simple où un seul type de site de chimisorption existe. L'intensité se réduit alors au produit d'un facteur de forme $\left|K\left(k_{2}^{-} \leftarrow k_{1}^{+}\right)\right|^{2}$ par un facteur de structure $G\left(k_{2 \|}-k_{1 \|}\right)$. L'information contenue dans le facteur de forme peut être isolée de celle contenue dans le facteur de structure en calculant la dérivée logarithmique de l'intensité diffusée à $k_{2 \|}-k_{1 \|}$ constant $[15,16]$. En fait, cette dérivée logarithmique $L$ n'est pas directement utilisée car elle donne un poids trop important au région du spectre où l'intensité est petite. La comparaison expérience/théorie est effectuée en utilisant une fonction notée $Y=L^{-1} /\left(L^{-2}+V_{\mathrm{a}}\right), V_{\mathrm{a}}$ étant le potentiel d'adsorption du substrat [16]. Les fonctions $Y$ expérimentale et théorique sont comparées en utilisant un facteur de confiance $R=\left(Y_{\mathrm{e}}-Y_{\mathrm{t}}\right)^{2} /\left(Y_{\mathrm{e}}+Y_{\mathrm{t}}\right)^{2}$ [16]. Cette méthode peut donc être appliquée dans un premier temps à la recherche d'un bon facteur forme de l'adsorbat. Les propriétés de diffusion d'un atome adsorbé sont généralement connues: les paramètres qui les caractérisent sont les déphasages atomiques de cet atome. Les seuls paramètres à ajuster pour minimiser $R$ sont donc ceux caractérisant la géométrie du site de chimisorption. Une fois obtenu un jeu optimum de ces paramètres, donc un bon facteur de forme, on peut dans un deuxième temps rechercher le facteur de structure $G\left(k_{2 \|}-k_{1 \|}\right)$ et par transformée de Fourier inverse, la fonction de corrélation de paire réduite.

Dans la situation où plusieurs types de sites d'adsorption existent, l'intensité rétrodiffusée ne se réduit plus au produit d'un facteur de forme par un facteur de structure et la méthode de la fonction $Y$ ne peut plus être appliquée. L'ajustement expérience/théorie est alors effectué en comparant directement les intensités mesurées et calculées. Si la couche adsorbée est totalement désordonnée, $\gamma_{p p^{\prime}}\left(\boldsymbol{R}_{i}\right)=0$ pour $\boldsymbol{R}_{i} \neq 0$. Dans le cas où deux adsorbats ne peuvent coexister dans une maille du réseau, $\gamma_{p p^{\prime}}(0)=-\theta_{p} \theta_{p^{\prime}}$ si $p \neq p^{\prime}$ et $\gamma_{p p}(0)=\theta_{p}\left(1-\theta_{p}\right)$ [14]. Les seuls paramètres à ajuster sont donc ceux caractérisant la géomètrie des sites de chimisorption et les taux de couverture partiels $\theta_{p}$. Si la couche est partiellement ordonnée, il faut alors calculer les fonctions de corrélation de la couche adsorbée. Ce type de calcul, qui peut être effectué par différentes méthodes (méthodes des cummulants, du champ moyen, de Monte Carlo), nécessite la connaissance des interactions entre adsorbats [17].

Pour terminer, nous signalerons que l'adsorption d'un atome induit souvent de légers déplacements $\delta \boldsymbol{R}$ des atomes du substrat appartenant à son site d'adsorption. Une méthode, dite du "tensor LEED" [18], a été proposée pour tenir compte de cette reconstruction locale du substrat: les déplacements $\delta \boldsymbol{R}$ sont généralement assez petits, pour que la matrice de diffusion multiple $M_{S}$ du substrat puisse être développée en série de puissances de $\delta \boldsymbol{R}$. Les coefficients de cette série sont de nature tensorielle, d'où le nom de la méthode. Une fois ces coefficients calculés, l'amplitude de diffusion du substrat relaxé ainsi que les matrices de transition renormalisées en présence du substrat relaxé peuvent être rapidement calculées. L'ajustement expérience/théorie qui inclus, en plus de la position 
des atomes de la maille, les relaxations du substrat, peut alors être réalisé avec des temps de calcul raisonnable.

\section{Références}

[1] P. Lloyd, P.V. Smith, Adv. Phys. 21, 69 (1972).

[2] K. Kambe, Z. Nat.forsch. 22a, 322 (1967).

[3] K. Kambe, Z. Nat.forsch. 22b, 422 (1967).

[4] K. Kambe, Z. Nal.forsch. 23a, 1280 (1968).

[5] J.B. Pendry, Low Energy Electron Diffraction, Academic Press, London 1974.

[6] E.G. Mc Rae, Surf. Sci. 11, 479 (1968).

[7] J.C. Le Bossé, J. Lopez, J. Rousseau, I. Zasada, J. Phys., Condens. Matter 4, 1671 (1992).

[8] K. Heinz, K. Muller, in: LEED Intensilies - Experimental Progress and New Possibilities in Surface Structure Determination, Springer Tracts of Modern Physics, Vol. 91, Ed. G. Hohler, Springer, Berlin 1982.

[9] I. Zasada, Thèse de doctorat, Université C. Bernard-Lyon I, 1992.

[10] J.B. Pendry, D.K. Saldin, Surf. Sci. 145, 33 (1984).

[11] D.K. Saldin, J.B. Pendry, Comput. Phys. Commun. 42, 399 (1986).

[12] D.K. Saldin, J.B. Pendry, Comput. Phys. Commun. 46, 129 (1987).

[13] J.C. Le Bossé, L. Lopez, J. Rousseau, I. Zasada, J. Phys. C, Solid State Phys. 21, $565(1988)$.

[14] J.C. Le Bossé, L. Lopez, J. Rousseau, I. Zasada, L. Wojtczak, J. Phys., Condens. Malter 2, 3143 (1990).

[15] K. Heinz, D.K. Saldin, J.B. Pendry, Phys. Rev. Lett. 55, 2312 (1985).

[16] J.B. Pendry, J. Phys. C, Solid State Phys. 13, 937 (1980).

[17] G. Wiatrowski, J.C. Le Bossé, J. Lopez, I. Zasada, Surf. Sci. 265, 229 (1992).

[18] P.J. Rous, J.B. Pendry, D.K. Saldin, K. Heinz, K. Müller, N. Bickel, Phys. Rev. Lett. 57, 2951 (1986). 